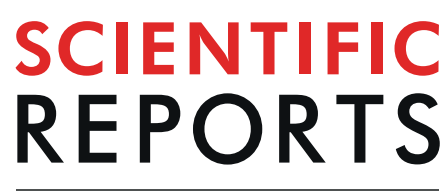

natureresearch

Check for updates

\title{
Inappropriate Peak Inspiratory Flow Rate with Dry Powder Inhaler in Chronic Obstructive Pulmonary Disease
}

\begin{abstract}
Shih-Yu Chen ${ }^{1}$, Chun-Kai Huang ${ }^{1,2}$, Hui-Chuan Peng ${ }^{3}$, Chong-Jen Yu $^{1}$ \& Jung-Yien Chien ${ }^{1 凶}$
Optimal peak inspiratory flow rate (PIFR) is crucial for optimizing dry powder inhaler (DPI) effectiveness for chronic obstructive pulmonary disease (COPD). This study provide an insight that there was a substantial proportion of improper PIFRs (not only insufficient but also excessive) among COPD patients using DPIs. We enrolled 138 COPD patients from a medical center in Taiwan and measured PIFRs against different internal resistances of DPIs. Proportion of excessive, optimal, suboptimal, and insufficient PIFRs were $2 \%, 54 \%, 41 \%, 3 \%$, respectively, against medium-high resistance; $2 \%, 77 \%, 20 \%, 1 \%$, respectively, against medium resistance; $27 \%, 63 \%, 9 \%, 1 \%$, respectively, against medium-low resistance; and $42 \%, 57 \%, 1 \%, 0 \%$, respectively, against low resistance $(p<0.01)$. Although most PIFRs against medium-high (54\%), medium (77\%), medium-low (63\%) and low (57\%) resistance were optimal, a substantial proportion of PIFRs against low resistance were excessive $(42 \%, p<0.01)$, irrespective of age, body-mass index, dyspnea severity score, and COPD severity. Insufficient PIFRs were infrequent, but suboptimal/insufficient PIFRs were most prevalent in patients older than 75 years than in younger patients ( $36 \%$ vs. $56 \%, p=0.036$ ) against medium-high resistance. Regularly monitoring PIFRs against the specific resistance of the DPIs and instructing patients to employ a proper inspiration effort may help to optimize the effects of DPIs.
\end{abstract}

Dry powder inhalers (DPIs), a breath-actuated inhalation systems, required patients to generate sufficient inspiratory flow and turbulence in the device to disaggregate the powder into fine particles ${ }^{1,2}$. Thus, it is generally advised to inhale with a forced inspiratory maneuver to generate adequate peak inspiratory flow rate (PIFR) to overcome the internal resistance of the devices ${ }^{1-5}$. The PIFR is impacted by several factors, such as sex, age, height, the internal resistance of DPIs, inhalation effort, pulmonary function, and even the period following acute exacerbation due to chronic obstructive pulmonary disease $(\mathrm{COPD})^{6-8}$. It is generally suggested that PIFR less than $30 \mathrm{~L} / \mathrm{min}$ is insufficient and the suggested optimal PIFR is at least $60 \mathrm{~L} / \mathrm{min}$ for Turbuhaler, Ellipta and Accuhaler, and $50 \mathrm{~L} /$ $\min$ for Breezhaler ${ }^{5,6}$. However, compared to optimal PIFR, excessive PIFR also lead to more oropharyngeal deposition and less lung deposition and a PIFR more than $90 \mathrm{~L} / \mathrm{min}$ was considered excessive ${ }^{9,10}$.

Different DPIs have different internal resistances, which can be categorized to medium-high, medium, medium-low and low internal resistances ${ }^{11}$. We aim to investigate the prevalence of improper PIFRs and the influencing factors against different internal resistances of DPIs, among COPD patients with varying disease severity.

\section{Methods}

Adult patients with stable COPD, who receiving medical treatment in our outpatient clinics without acute exacerbation during previous 3 months, were recruited from National Taiwan University Hospital from May 2017 to February 2019. The patients were diagnosed according to the GOLD criteria defined by $<70 \%$ post-bronchodilator forced expiratory volume in one second (FEV1) to forced vital capacity (FVC) ratio (FEV1/ FVC ratio). Data on patients' demographics, results of pulmonary function tests, smoking status, dyspnea severity classified by scores of modified medical research council (mMRC) and COPD assessment test (CAT) were

${ }^{1}$ Department of Internal Medicine, National Taiwan University Hospital, College of Medicine, National Taiwan University, Taipei, Taiwan. ${ }^{2}$ Institute of Epidemiology and Preventive Medicine, College of Public Health, National Taiwan University, Taipei, Taiwan. ${ }^{3}$ Department of Nursing, National Taiwan University Hospital, College of Medicine, National Taiwan University, Taipei, Taiwan. ${ }^{\circledR e}$-mail: jychien@ntu.edu.tw 


\begin{tabular}{|c|c|c|c|c|c|c|}
\hline \multirow[b]{2}{*}{ Variable } & \multirow[b]{2}{*}{ All } & \multicolumn{4}{|l|}{ GOLD Group } & \multirow[b]{2}{*}{ p Value } \\
\hline & & Group $A(n=36)$ & Group $B(n=75)$ & Group $C(n=7)$ & Group $\mathrm{D}(\mathrm{n}=\mathbf{2 0})$ & \\
\hline Age in years & $72(37-91)$ & $71(37-87)$ & $72(54-85)$ & $79(65-82)$ & $73(56-87)$ & 0.406 \\
\hline Male & $131(95)$ & $32(89)$ & $73(97)$ & $6(86)$ & $20(100)$ & 0.099 \\
\hline Weight, kg & $63(40-102)$ & $63.2(43-87)$ & $62.9(40-102)$ & $56(49-71)$ & $66(52-81)$ & 0.319 \\
\hline Height, cm & $164(146-180)$ & $162(147-180)$ & $162.8(146-180)$ & $165(158-170)$ & $164.5(158-172)$ & 0.567 \\
\hline Body mass index, $\mathrm{kg} / \mathrm{m}^{2}$ & $23.2(16-36.1)$ & $22.85(19.4-29.1)$ & $23.7(16-36.1)$ & $20.6(19.1-21.6)$ & $24(19.3-28.6)$ & 0.289 \\
\hline FEV1, L & $1.46(0.45-3.25)$ & $1.79(0.75-3.25)$ & $1.35(0.45-2.54)$ & $1.46(0.85-2.32)$ & $1.36(0.83-2.44)$ & 0.009 \\
\hline FEV1, \% predicted & $71.8(22.3-131.1)$ & $80(34.5-130.9)$ & $69.2(22.3-131.1)$ & $75.7(54.9-89.8)$ & $63.6(32.3-124.4)$ & 0.014 \\
\hline Smoking status & & & & & & 0.576 \\
\hline Current smoker & $28(20)$ & $10(28)$ & $14(19)$ & $1(14)$ & $3(15)$ & \\
\hline Former smoker & $74(54)$ & $18(50)$ & $44(59)$ & $3(43)$ & $9(45)$ & \\
\hline Never smoker & $19(14)$ & $3(8)$ & $10(13)$ & $2(29)$ & $4(20)$ & \\
\hline mMRC dyspnea score & & & & & & $<0.001$ \\
\hline Grade 0 & $4(3)$ & $3(8)$ & $1(1)$ & 0 & 0 & \\
\hline Grade 1 & $43(31)$ & $33(92)$ & $3(4)$ & $7(100)$ & 0 & \\
\hline Grade 2 & $67(49)$ & 0 & $56(75)$ & 0 & $11(55)$ & \\
\hline Grade 3 & $24(17)$ & 0 & $15(20)$ & 0 & $9(45)$ & \\
\hline Grade 4 & 0 & 0 & 0 & 0 & 0 & \\
\hline CAT score & & & & & & $<0.001$ \\
\hline$\leq 10$ & $106(77)$ & $36(100)$ & $51(68)$ & $7(100)$ & $12(60)$ & \\
\hline $11-20$ & $29(21)$ & 0 & $23(31)$ & 0 & $6(30)$ & \\
\hline $21-30$ & $3(2)$ & 0 & $1(1)$ & 0 & $2(10)$ & \\
\hline $31-40$ & 0 & 0 & 0 & 0 & 0 & \\
\hline \multicolumn{7}{|l|}{ Comorbidity } \\
\hline $\begin{array}{l}\text { Cerebrovascular accident } \\
\text { or neuromuscular disease }\end{array}$ & $12(9)$ & $1(3)$ & $10(13)$ & $1(14)$ & 0 & 0.120 \\
\hline Head and neck tumor & $5(4)$ & $2(6)$ & $3(4)$ & 0 & 0 & 0.698 \\
\hline Cardiovascular diseases & $50(36)$ & $7(19)$ & $31(41)$ & $2(29)$ & $10(50)$ & 0.070 \\
\hline Asthma & $13(9)$ & $2(6)$ & $7(9)$ & $1(14)$ & $3(15)$ & 0.670 \\
\hline Hypertension & $65(47)$ & $13(36)$ & $40(53)$ & 0 & $12(60)$ & 0.015 \\
\hline Diabetes mellitus & $36(26)$ & $8(22)$ & $17(23)$ & 0 & $4(20)$ & 0.566 \\
\hline
\end{tabular}

Table 1. Characteristics of patients with chronic obstructive pulmonary disease. Data presented as $\mathrm{n}(\%)$ or median (range). CAT, Chronic obstructive pulmonary disease assessment test; FEV1, forced expiratory volume in one second; GOLD: Global Initiative for Chronic Obstructive; mMRC, modified medical research council.

recorded. According to GOLD guideline, we classified COPD patients into GOLD Group ABCD according to frequency of exacerbation and mMRC or CAT scoring system with the greater score. PIFRs were measured against four-degrees of internal resistances (low, medium-low, medium, and medium-high) using the In-Check Dial G16 (Clement-Clarke International Ltd, Harlow, UK), a handheld inspiratory flow device simulates different internal resistances of DPIs ${ }^{11}$. For low resistance devices, measured PIFRs were classified to excessive $(\geq 100 \mathrm{~L} /$ $\mathrm{min}$ ), optimal (50-99L/min), suboptimal (30-49L/min), and insufficient $(<30 \mathrm{~L} / \mathrm{min})^{12}$, and for those other than low resistance devices, measured PIFRs were classified into four categories, excessive $(\geq 90 \mathrm{~L} / \mathrm{min})$, optimal $(60-89 \mathrm{~L} / \mathrm{min})$, suboptimal $(30-59 \mathrm{~L} / \mathrm{min})$, and insufficient $(<30 \mathrm{~L} / \mathrm{min})^{5,6,10}$. The institution board of National Taiwan University Hospital (201905058RINB) approved this study and written informed consent was waived by the ethics committee due to the retrospective nature of the study. All methods were carried out in accordance with relevant guidelines and regulations.

Statistical analysis. Categorical variables were compared using a chi-square test or Fisher's exact test, as appropriate. Differences in continuous variables were analyzed using the Mann-Whitney U test or ANOVA. The data are presented as numbers (percentages), median (range), mean \pm standard deviation, and $\mathrm{p}=0.05$ is considered a statistically significant difference. Linear regression analysis was used to investigate the relationship between PIFR and FEV1, FVC, age and body height. The statistical analyses were performed using STATA version 14 software (StataCorp LLC, TX).

\section{Results}

A total of 138 patients with stable COPD underwent PIFR measurement during the study. The median age was 72 (37-91) years, and most participants were men $(131,94.9 \%)$. The median height was $164(146-180) \mathrm{cm}$ while the median weight was $63(40-102) \mathrm{kg}$. Mean forced expiratory volume in one second (FEV1) was $1.53 \pm 0.49 \mathrm{~L}$, and the mean percentage of predicted value was $70.9 \pm 21.8 \%$. Table 1 shows the demographic parameters.

The median PIFR was 63 (21-98) L/min against medium-high, 71 (25-103) L/min against medium, 80 (26116) L/min against medium-low, and 97 (34-150) L/min against low resistances. Measured PIFRs positively 


\section{Med-high resistance}

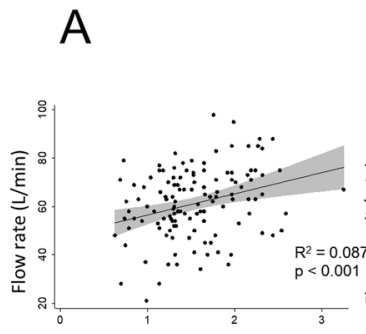

FEV1 (L), post-bronchodilator

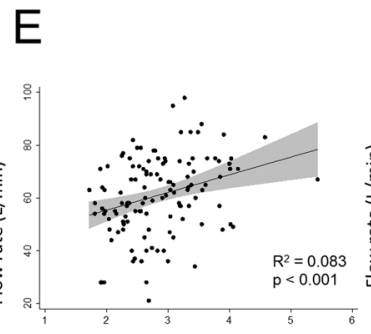

FVC (L),post-bronchodilator

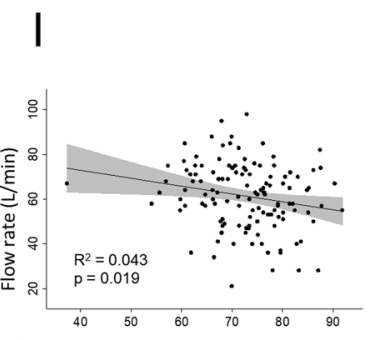

Age (years)
M

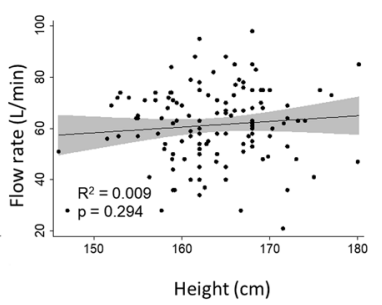

\section{Medium resistance}

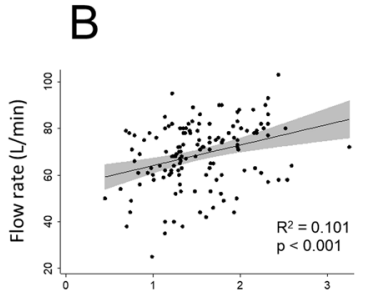

FEV1 (L), post-bronchodilator

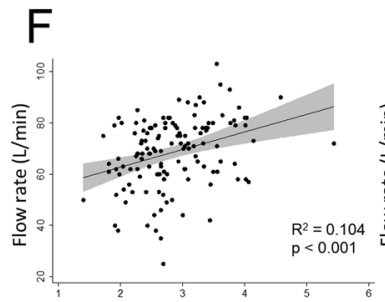

FVC (L),post-bronchodilator

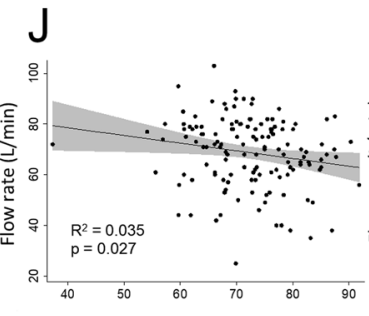

Age (years)

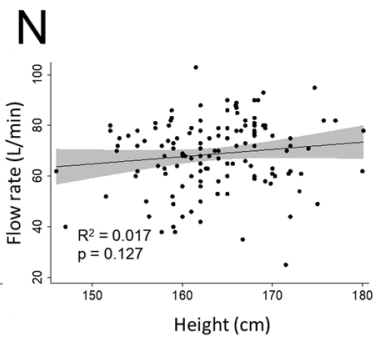

Med-low resistance
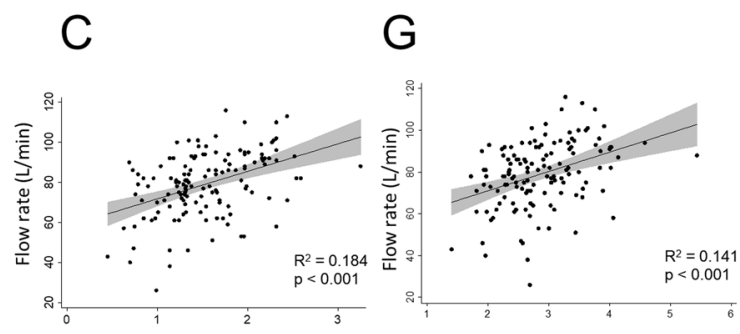

FVC (L),post-bronchodilator

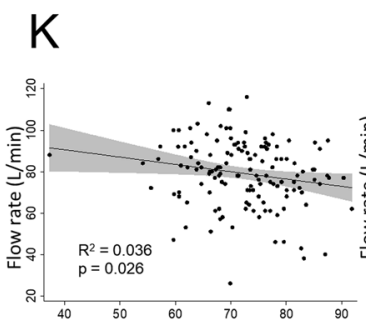

Age (years)

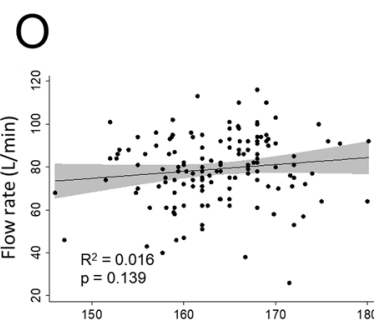

Height $(\mathrm{cm})$

\section{Low resistance}

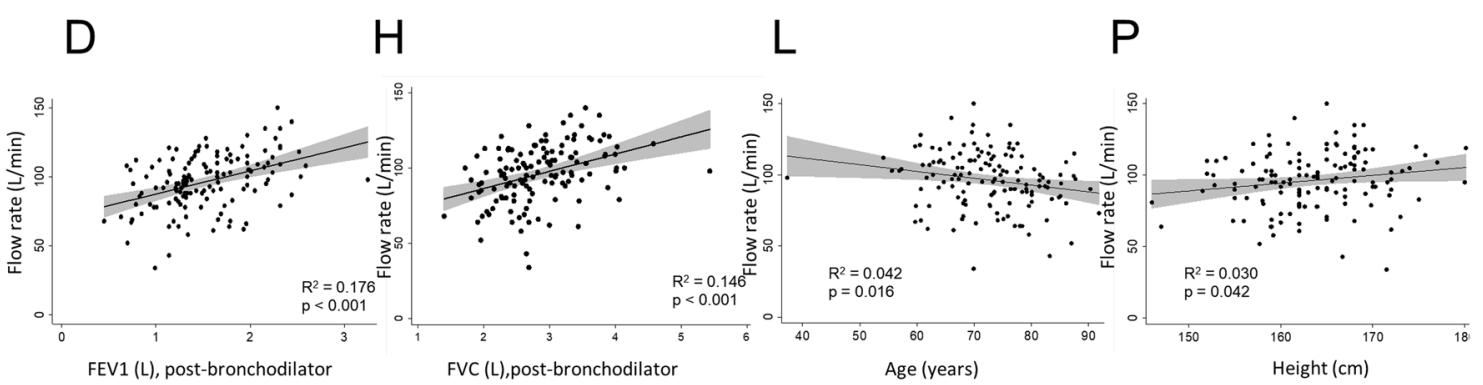

Figure 1. Scatter plot and regression line between peak inspiratory flow rate and forced expiratory volume in one second (FEV1, Panel A-D), percent predicted value of forced vital capacity (FVC\%, Panel E-H), age (Panel $\mathrm{I}-\mathrm{L}$ ) and body height (Panel M-P) against different simulated internal resistances of dry powder devices.

correlated with FEV1 and forced vital capacity (FVC) but negatively correlated with age and didn't correlate with body height (Fig. 1). The percentage of insufficient, suboptimal, optimal and excessive PIFRs against low resistance were $0 \%(n=0), 1 \%(n=2), 57 \%(n=78), 42 \%(n=58)$, respectively, against medium-low resistance were $1 \%(\mathrm{n}=1), 9 \%(\mathrm{n}=13), 63 \%(\mathrm{n}=87), 27 \%(\mathrm{n}=37)$, respectively, and against medium resistance were $1 \%(\mathrm{n}=1)$, $20 \%(n=28), 77 \%(n=106), 2 \%(n=3)$, respectively $(p<0.01)$. Among 127 patients, PIFRs measured against medium-high resistance of DPIs, 4 (3\%) were insufficient, 52 (41\%) were suboptimal, 69 (54\%) were optimal, 


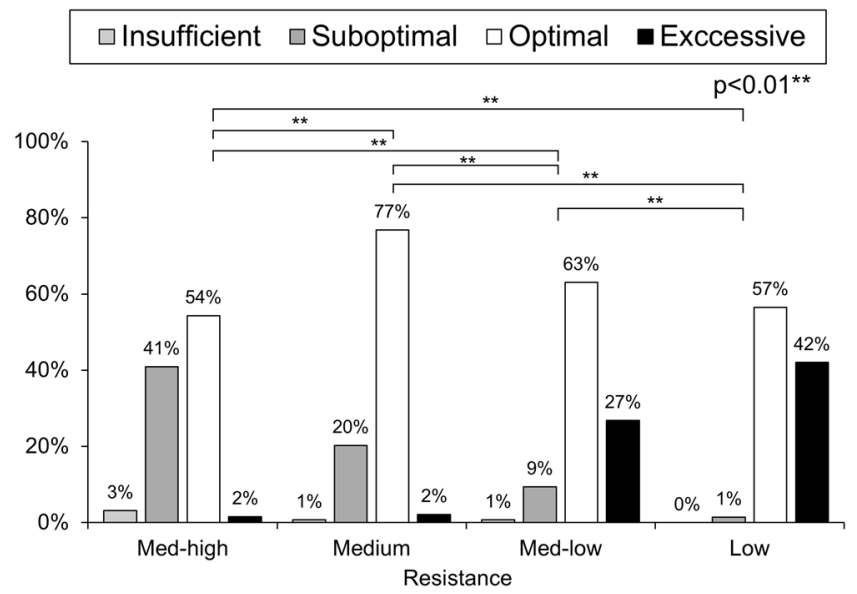

Figure 2. Prevalence of excessive, optimal, suboptimal, and insufficient peak inspiratory flow rates measured against different resistances $(\mathrm{p}<0.01)$. ** represents $\mathrm{p}<0.01$ between each resistance.

\begin{tabular}{|c|c|c|c|c|}
\hline \multirow[b]{2}{*}{ Variable } & \multicolumn{4}{|c|}{ Internal resistance } \\
\hline & Med-high & Medium & Med-low & Low \\
\hline \multicolumn{5}{|l|}{ Age, years } \\
\hline$<65$ & $68(36-85)$ & $75.5(44-95)$ & $84(47-103)$ & $103.5(62-128)$ \\
\hline $65-70$ & $63.5(21-95)$ & $72(25-103)$ & $80(26-113)$ & $100(34-150)$ \\
\hline $70-75$ & $64.5(40-98)$ & $74.5(46-90)$ & $86(53-116)$ & $103.5(66-128)$ \\
\hline $75-80$ & $57(28-85)$ & $70(38-86)$ & 75 (46-98) & $93(58-135)$ \\
\hline$\geq 80$ & $58(28-82)$ & $65.5(35-83)$ & $76.5(38-95)$ & $91.5(43-115)$ \\
\hline $\mathrm{p}$ value & 0.126 & 0.204 & 0.188 & 0.124 \\
\hline \multicolumn{5}{|c|}{ Body mass index, $\mathrm{kg} / \mathrm{m}^{2}$} \\
\hline$<18.5$ & $70.5(52-79)$ & $76(56-80)$ & $86(69-95)$ & $104(78-112)$ \\
\hline $18.5-24$ & $59(21-88)$ & $66.5(25-90)$ & $75(26-101)$ & $95(34-150)$ \\
\hline $24-27$ & $66(28-95)$ & $72(35-103)$ & $82.5(38-113)$ & $102(43-140)$ \\
\hline$\geq 27$ & $62(34-98)$ & $74(42-95)$ & $86(51-116)$ & $103(61-122)$ \\
\hline $\mathrm{p}$ Value & 0.283 & 0.068 & 0.036 & 0.300 \\
\hline \multicolumn{5}{|l|}{ Sex } \\
\hline Man & $63(21-98)$ & $71(25-103)$ & $81(26-116)$ & $98(34-150)$ \\
\hline Woman & $56(28-74)$ & $60(40-75)$ & $73(46-88)$ & $89(64-110)$ \\
\hline $\mathrm{p}$ Value & 0.171 & 0.04 & 0.077 & 0.182 \\
\hline \multicolumn{5}{|l|}{ mMRC } \\
\hline Grade 0 & $46(28-62)$ & $51.5(40-76)$ & $57.5(46-91)$ & $70(64-113)$ \\
\hline Grade 1 & $64(28-98)$ & $72(38-103)$ & $84(40-116)$ & $97(52-140)$ \\
\hline Grade 2 & $60.5(21-95)$ & 70 (25-95) & $78(26-110)$ & $97(34-135)$ \\
\hline Grade 3 & $63(34-88)$ & $70.5(38-90)$ & $82(46-103)$ & $102.5(58-150)$ \\
\hline $\mathrm{p}$ Value & 0.138 & 0.140 & 0.157 & 0.275 \\
\hline \multicolumn{5}{|c|}{ COPD GOLD stage } \\
\hline GOLD 1 & $62.5(28-98)$ & $71.5(38-90)$ & $82.5(40-116)$ & $96.5(52-135)$ \\
\hline GOLD 2 & $61.5(21-95)$ & $70(25-103)$ & $78(26-113)$ & $97(34-140)$ \\
\hline GOLD 3 & $62.5(34-85)$ & $67.5(38-86)$ & $79.5(46-103)$ & $101(58-122)$ \\
\hline GOLD 4 & $70(68-88)$ & $86(74-90)$ & $98(86-101)$ & $122(103-150)$ \\
\hline $\mathrm{p}$ Value & 0.348 & 0.184 & 0.378 & 0.083 \\
\hline \multicolumn{5}{|c|}{ COPD GOLD group } \\
\hline Group A & $62.5(28-98)$ & $72(40-90)$ & $85(46-116)$ & $97(64-135)$ \\
\hline Group B & $61.5(21-95)$ & $70(25-103)$ & $78(26-113)$ & $97(34-140)$ \\
\hline Group C & $60(34-84)$ & $65(38-86)$ & $70(46-102)$ & $91(58-122)$ \\
\hline Group D & $63.5(40-88)$ & $71(44-90)$ & $82(61-103)$ & $103(68-150)$ \\
\hline $\mathrm{p}$ Value & 0.758 & 0.736 & 0.638 & 0.400 \\
\hline
\end{tabular}

Table 2. Forced peak inspiratory flow rates against different simulated internal resistances of dry powder inhalers. Data presented as median (range). GOLD: Global Initiative for Chronic Obstructive; mMRC, modified medical research council. 

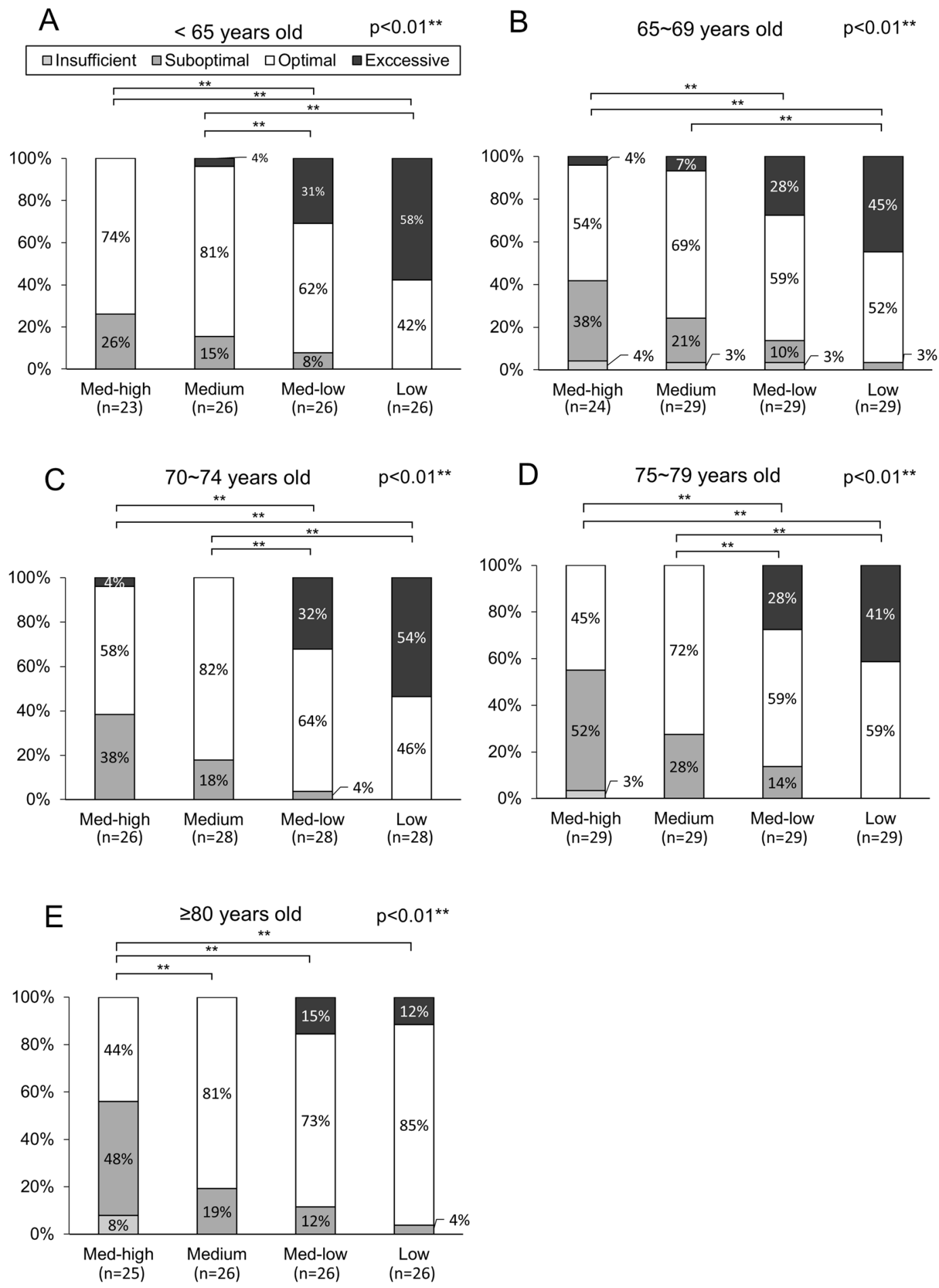

Figure 3. Percentage of excessive, optimal, suboptimal and insufficient peak inspiratory flow rates measured against different resistances among patients $<65$ (Panel A, p < 0.01), 65-69 (Panel B, p $<0.01$ ), 70-74 (Panel C, $\mathrm{p}<0.01$ ), 75-79 (Panel D, $\mathrm{p}<0.01$ ) and $\geq 80$ years of age (Panel $\mathrm{E}, \mathrm{p}<0.01$ ). * represents $\mathrm{p}<0.05$, ** represents $\mathrm{p}<0.01$.

and $2(2 \%)$ were excessive, respectively (Fig. $2, \mathrm{p}<0.01$ ). Further subgroup analysis in Table 2 and Figs. $3-5$ show that regardless of age groups, gender, body mass index (BMI), severity of dyspnea by mMRC score, GOLD stages or GOLD groups, there were more optimal PIFRs as measured against medium internal resistance, while there was a majority of excessive PIFRs as measured against low resistance and more suboptimal PIFRs as measured against medium-high resistance. Figure 3 also shows that patients $>75$ years have a higher prevalence of suboptimal or insufficient PIFRs than younger patients $(36 \%$ vs. $56 \%, \mathrm{p}=0.036)$ when measured against DPIs with medium-high internal resistance. 
A
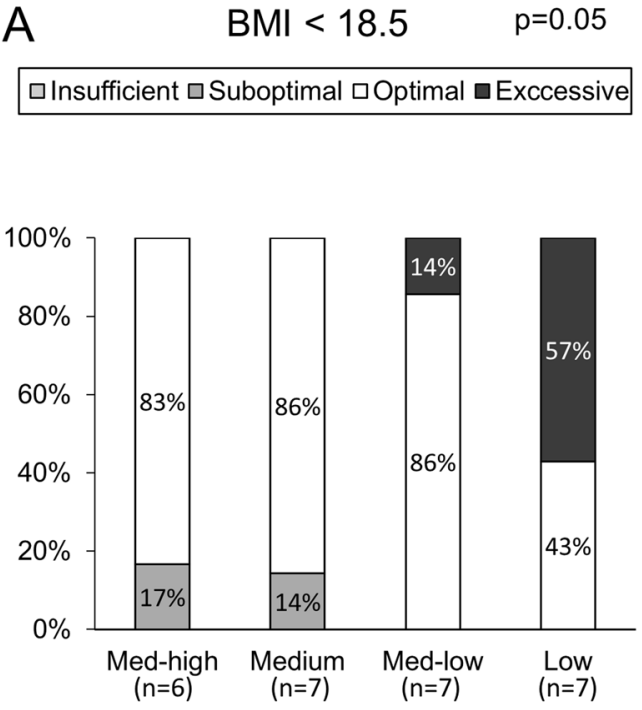

C
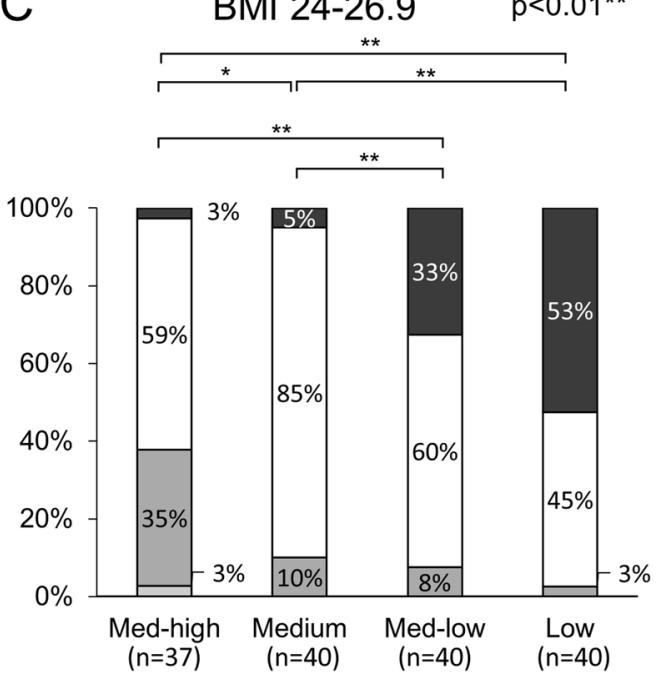

B

BMl 18.5-23.9 $p<0.01^{* *}$
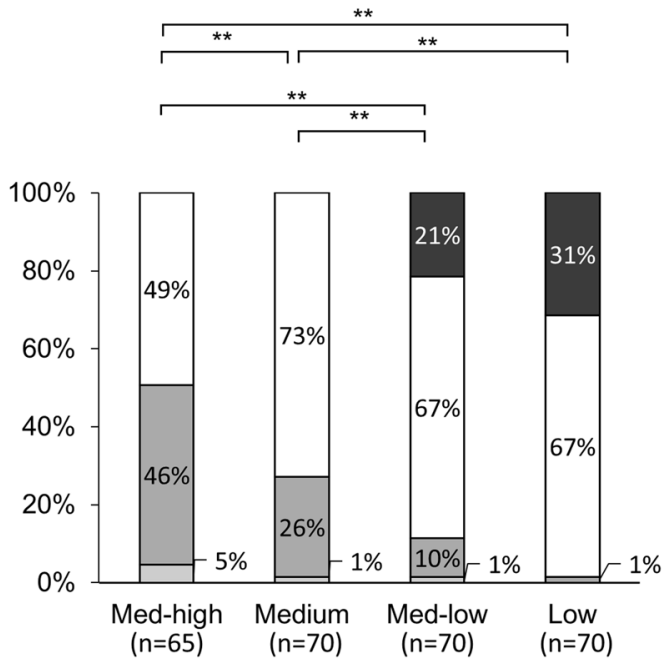

$\mathrm{D}$
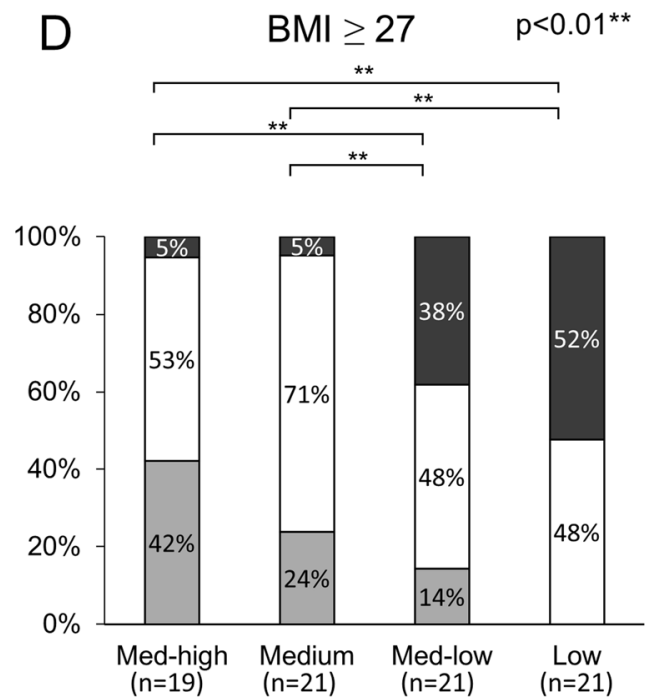

Figure 4. Percentage of excessive, optimal, suboptimal and insufficient peak inspiratory flow rates measured against different resistances among patients with body mass index $<18.5$ (Panel A, p $<0.01$ ), 18.5-23.9 (Panel $\mathrm{B}, \mathrm{p}<0.01$ ), 24-26.9 (Panel C, $\mathrm{p}<0.01$ ) and $\geq 27 \mathrm{~kg} / \mathrm{m}^{2}$ (Panel $\mathrm{D}, \mathrm{p}<0.01$ ). * represents $\mathrm{p}<0.05$; ** represents $\mathrm{p}<0.01$.

\section{Discussion}

We investigated the PIFRs against different internal resistances of DPIs among stable COPD patients and found correlations between PIFRs and FEV1, FVC, and age. Moreover, among stable COPD patients, we found that more PIFRs measured against medium resistance were optimal, more PIFRs against medium-high resistance were suboptimal, while a majority of PIFRs against low resistance were excessive.

Forced inspiration could provide faster acceleration rates, which increases the deaglommeration of particles before the dose leaves the device. Therefore, the guideline of inhalation therapies suggests "instruct the patient to inhale forcefully from the beginning" . This forced inspiration method provides a simple and precise instruction to the patients. This method is easy to remember, readily found in most prescribing information for DPIs and allows patients to perform the inhalation consistently in daily life ${ }^{5}$. More importantly, without the maximum inhalation effort, the relationship between the flow rate and the pressure drop will be inconsistent ${ }^{4}$. A PIFR between 60 and $90 \mathrm{~L} / \mathrm{min}$ was suggested as optimal by several previous studies ${ }^{13-15}$, and $30 \mathrm{~L} / \mathrm{min}$ is considered the minimum effective PIFR, making the range of $30-60 \mathrm{~L} / \mathrm{min}$ a debatable area ${ }^{6,10,11,16}$. Thus, in this study, this flow range of $30-60 \mathrm{~L} / \mathrm{min}$ was classified as suboptimal while those less than $30 \mathrm{~L} / \mathrm{min}$ as insufficient. Pavkov et al. studied 26 patients using Breezhaler, a low resistance device and found that a consistent fine particle mass can be achieved at rate of $50-100 \mathrm{~L} / \mathrm{min}$. In this way, the flow range of $50-100 \mathrm{~L} / \mathrm{min}$ was classified as optimal while $30-49 \mathrm{~L} / \mathrm{min}$ as suboptimal for low resistance devices ${ }^{12}$. High prevalence of suboptimal PIFRs were reported in previous studies which accounts for approximately $20-78 \%$ of studied population ${ }^{5,13,17-21}$. Similarly, our study 
A

GOLD Group A $p<0.01^{\text {** }}$

E

GOLD 1

$p<0.01^{* *}$

口Insufficient 口Suboptimal $\square$ Optimal घExccessive
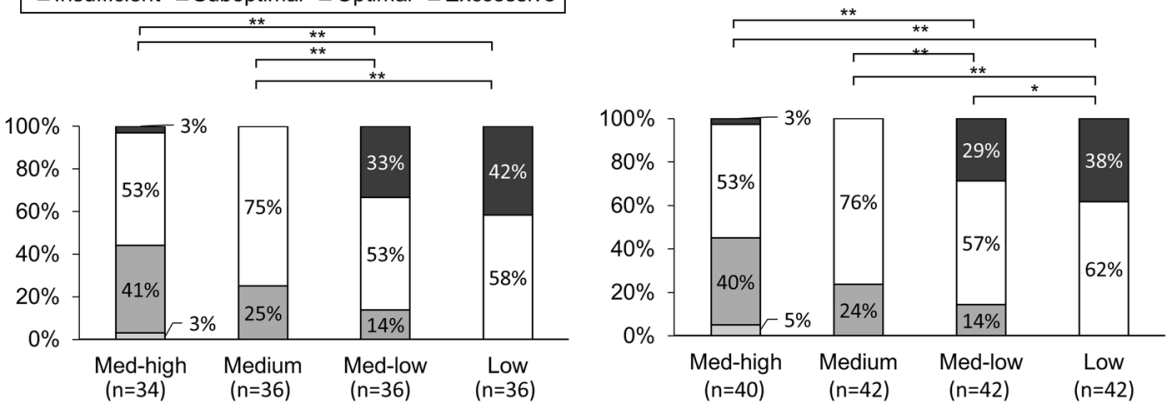

B

GOLD Group B $\quad p<0.01^{* *}$

$\mathrm{F}$
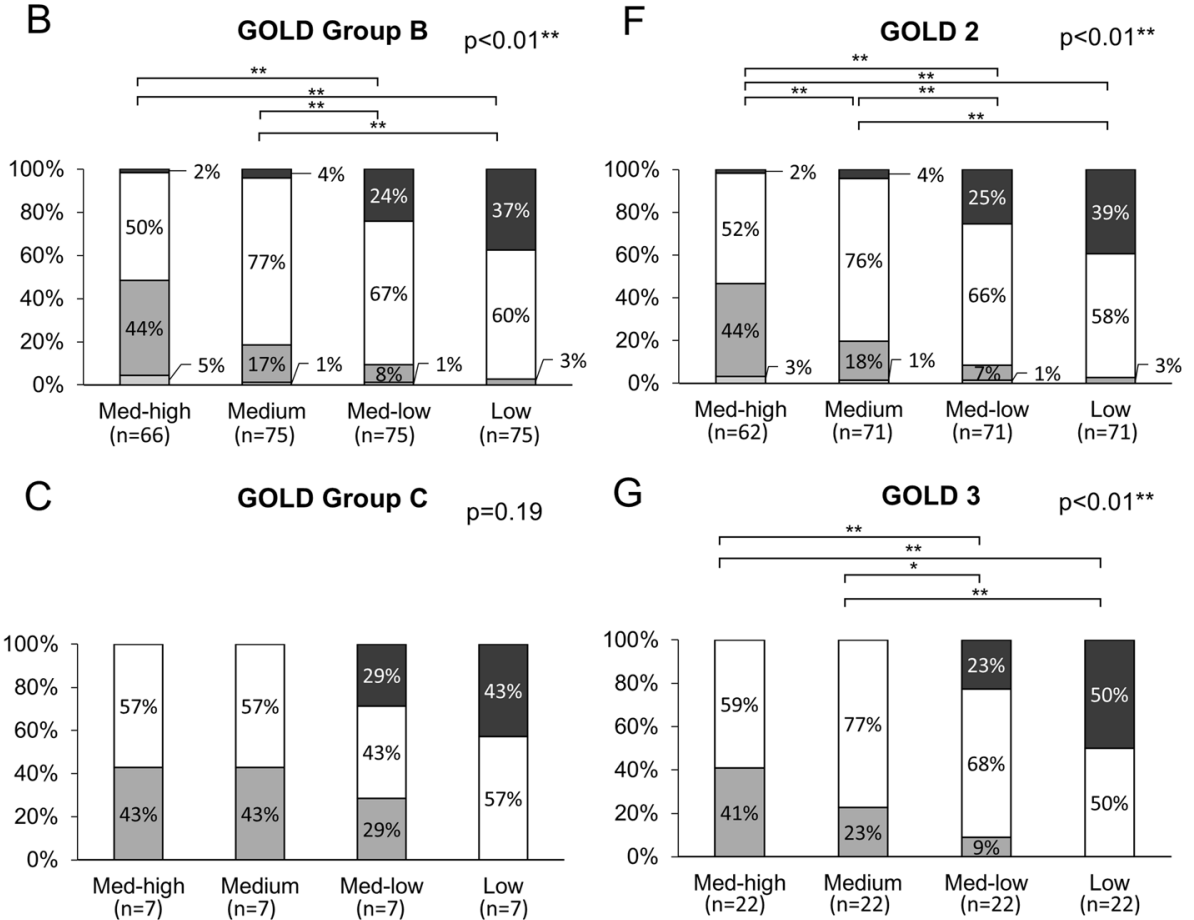

$\mathrm{D}$

GOLD Group D $\quad p<0.01^{* *}$

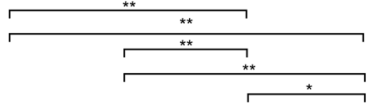

$\mathrm{H}$

GOLD 4

$p=0.25$
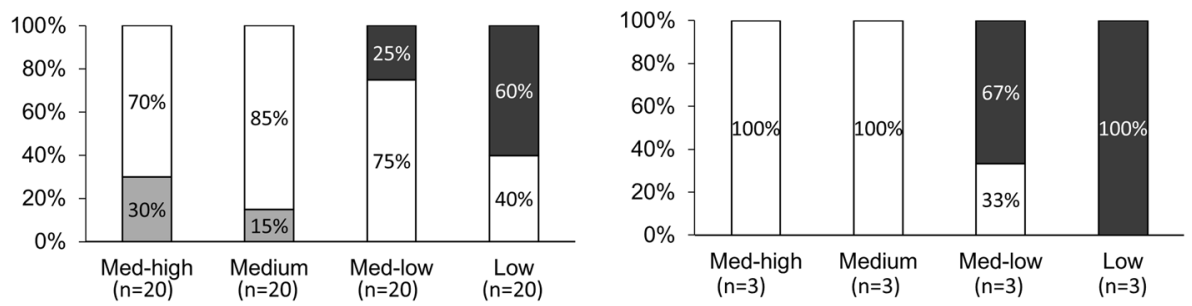

Figure 5. Percentage of excessive, optimal, suboptimal and insufficient peak inspiratory flow rates measured against different resistances among patients with Global Initiative for Chronic Obstructive Lung Disease (GOLD) group A (Panel A, $\mathrm{p}<0.01$ ), group B (Panel B, $\mathrm{p}<0.01$ ), group C (Panel C, $\mathrm{p}=0.22$ ), group D (Panel $\mathrm{D}, \mathrm{p}<0.01$ ) and GOLD stage 1 (Panel E, $\mathrm{p}<0.01$ ), stage 2 (Panel F, $\mathrm{p}<0.01$ ), stage 3 (Panel $\mathrm{G}, \mathrm{p}<0.01$ ) and stage $4($ Panel $\mathrm{H}, \mathrm{p}=0.045)$. represents $\mathrm{p}<0.05$; ** represents $\mathrm{p}<0.01$.

found the proportion of suboptimal or insufficient PIFRs range from 3-44\% among stable COPD patients with different severity.

However, although most studies emphasized on suboptimal PIFRs as a major problem of inappropriate DPI usage, we further found a substantial proportion of PIFRs were excessive when measured against medium-low 
and low resistance (27\% and $42 \%$, respectively). Excessive inspiratory flow rates also have negative impact on drug deposition in respiratory tract ${ }^{2}$. Usmani et al. used inhaled technetium-99m-labeled monodisperse albuterol aerosols and compared the respiratory deposition at slow and fast inspiratory flow rates, and found that faster inspiratory flows yield more oropharyngeal and central lung deposition (regardless of particle size), decreased total lung deposition, and lesser clinical effectiveness ${ }^{9}$. We found that patients tend to have excessive PIFRs as measured against medium-low or low resistance, while suboptimal or insufficient PIFR values resulted when measured against medium-high resistance. This remained true when patients were subdivided by BMI, age, COPD group or COPD stage, emphasizing that the prevalence of excessive or suboptimal peak inspiratory flow rates could be highly correlated with an internal resistance of DPIs per se.

Several pulmonary function parameters were also found to have association with PIFRs. Mahler et al. measured PIFR against medium-low resistance in COPD GOLD stage 3 and 4 patients (mean FEV1 of $0.92 \pm 0.26 \mathrm{~L}$ ) and found that PIFRs are associated with FVC and inspiratory capacity ${ }^{19}$. Duates et al. demonstrated a significant correlation between PIFRs and severity of air trapping, represented by the ratio of residual volume over total lung capacity (RV/TLC) ${ }^{22}$. We found values of PIFRs correlated positively with FEV1 and FVC. This finding was different from Janssens et al. where PIFRs were not statistically correlated with FEV1. This possibly is because they measured PIFRs against zero resistance and the smaller study population (26 COPD patients) in their study ${ }^{13}$.

Similar to previous studies ${ }^{13,18-20}$, we found a negative correlation between age and PIFRs $(\mathrm{p}<0.05)$. Furthermore, as measured against medium-high resistance, patients more than 75 years have a higher prevalence of suboptimal or insufficient PIFR ( $36 \%$ vs. $56 \%, p=0.036)$. In contrast, the prevalence of suboptimal or insufficient PIFRs did not significantly correlate with age among medium, medium-low, and low resistance of DPIs. It was similar to the study conducted by Kawamatawong et al. that older patients had a higher proportion of suboptimal PIFR breathing against Turbuhaler than Accuhaler (19.3\% vs 9.3\%) $)^{18}$.

There are some limitations to our study. First, our COPD population is predominantly male, which may not be representative of the spectrum of the COPD population worldwide, although our papulation was similar to a random cross-sectional national survey in Taiwan which showed males accounted for $78.9 \%$ of the COPD population $^{23}$. However, this male predominant papulation could lead to female gender and short stature, two important predictors of reduced PIFRs in the previous studies, did not have significant roles in this study ${ }^{17,19,20,22,24}$. Second, as patients may not exert maximal inspiratory effort every time in daily life, there may be a difference between in-office evaluation and daily practice at home. The very small number of participants with BMI $<18.5$ $(n=7)$, GOLD stage $4(n=3)$ and mMRC grade $0(n=4)$ substantially led to great variations and pseudo-higher PIFRs in patients with BMI $<18.5$ and GOLD stage 4 and pseudo-lower PIFRs in those with mMRC grade 0 (all the differences were not statistically significant). Therefore, there was great limitation and the interpretation of those findings should be very cautious. Fourth, this is a cross-sectional study and all patients had received effective treatment and pulmonary rehabilitation program without inspiratory muscle training for a period of time. A further prospective study design was needed in the future to address the effects of treatment and pulmonary rehabilitation on the PIFR. Finally, our study population is mainly stable COPD patients without acute exacerbation in previous 3 months. The result cannot be applied to those experiencing acute exacerbation of chronic obstructive disease or who just recover from acute illness.

Our study identified that PIFRs are correlated with FEV1, FVC, and age. We also revealed a substantial proportion of improper PIFRs (excessive and suboptimal) as measured against different resistances of DPIs, but insufficient PIFRs were infrequent $(<5 \%)$. The highest probability of optimal PIFR was measured against medium resistance. The lower the internal resistance, the higher the probability of excessive PIFR was noted, and suboptimal PIFRs were more often noticed when measured against higher internal resistance. Both excessive and suboptimal PIFRs may negatively impact drug deposition, therefore, regularly monitoring PIFR against the resistance of specific DPI and instructing patients to employ a proper inspiration effort may help to optimize the effects of DPIs.

Received: 12 January 2020; Accepted: 13 April 2020;

Published online: 29 April 2020

\section{References}

1. Laube, B. L. et al. What the pulmonary specialist should know about the new inhalation therapies. The European respiratory journal. 37(6), 1308-1331 (2011).

2. Ibrahim, M., Verma, R. \& Garcia-Contreras, L. Inhalation drug delivery devices: technology update. Med Devices (Auckl). 8, 131-139 (2015).

3. Ganderton, D. General factors influencing drug delivery to the lung. Respir Med. 91(Suppl A), 13-16 (1997).

4. Clark, A. R. \& Hollingworth, A. M. The relationship between powder inhaler resistance and peak inspiratory conditions in healthy volunteers-implications for in vitro testing. Journal of aerosol medicine: the official journal of the International Society for Aerosols in Medicine. 6(2), 99-110 (1993)

5. Mahler, D. A. Peak Inspiratory Flow Rate as a Criterion for Dry Powder Inhaler Use in Chronic Obstructive Pulmonary Disease. Ann Am Thorac Soc. 14(7), 1103-1107 (2017).

6. Ghosh, S., Ohar, J. A. \& Drummond, M. B. Peak Inspiratory Flow Rate in Chronic Obstructive Pulmonary Disease: Implications for Dry Powder Inhalers. J Aerosol Med Pulm Drug Deliv. 30(6), 381-387 (2017).

7. Sharma, G. et al. Prevalence of Low Peak Inspiratory Flow Rate at Discharge in Patients Hospitalized for COPD Exacerbation. Chronic obstructive pulmonary diseases (Miami, Fla). 4(3), 217-224 (2017).

8. Magnussen, H. et al. Peak inspiratory flow through the Genuair inhaler in patients with moderate or severe COPD. Respir Med. 103(12), 1832-1837 (2009).

9. Usmani, O. S., Biddiscombe, M. F. \& Barnes, P. J. Regional lung deposition and bronchodilator response as a function of beta2agonist particle size. American journal of respiratory and critical care medicine. 172(12), 1497-1504 (2005).

10. Broeders, M. E., Molema, J., Vermue, N. A. \& Folgering, H. T. In Check Dial: accuracy for Diskus and Turbuhaler. Int J Pharm. 252(1-2), 275-280 (2003). 
11. Sanders, M. J. Guiding Inspiratory Flow: Development of the In-Check DIAL G16, a Tool for Improving Inhaler Technique. Pulmonary medicine. 2017, 1495867 (2017).

12. Pavkov, R. et al. Characteristics of a capsule based dry powder inhaler for the delivery of indacaterol. Curr Med Res Opin. 26(11), 2527-2533 (2010).

13. Janssens, W. et al. Inspiratory flow rates at different levels of resistance in elderly COPD patients. Eur Respir J. 31(1), 78-83 (2008).

14. Al-Showair, R. A., Tarsin, W. Y., Assi, K. H., Pearson, S. B. \& Chrystyn, H. Can all patients with COPD use the correct inhalation flow with all inhalers and does training help? Respiratory medicine. 101(11), 2395-2401 (2007).

15. Mahler, D. A., Waterman, L. A., Ward, J. \& Gifford, A. H. Comparison of dry powder versus nebulized beta-agonist in patients with COPD who have suboptimal peak inspiratory flow rate. J Aerosol Med Pulm Drug Deliv. 27(2), 103-109 (2014).

16. Chrystyn, H. Is inhalation rate important for a dry powder inhaler? Using the In-Check Dial to identify these rates. Respiratory medicine. 97(2), 181-187 (2003).

17. Ghosh, S., Pleasants, R. A., Ohar, J. A., Donohue, J. F. \& Drummond, M. B. Prevalence and factors associated with suboptimal peak inspiratory flow rates in COPD. Int J Chron Obstruct Pulmon Dis. 14, 585-595 (2019).

18. Kawamatawong, T., Khiawwan, S. \& Pornsuriyasak, P. Peak inspiratory flow rate measurement by using In-Check DIAL for the different inhaler devices in elderly with obstructive airway diseases. J Asthma Allergy. 10, 17-21 (2017).

19. Mahler, D. A., Waterman, L. A. \& Gifford, A. H. Prevalence and COPD phenotype for a suboptimal peak inspiratory flow rate against the simulated resistance of the Diskus(R) dry powder inhaler. J Aerosol Med Pulm Drug Deliv. 26(3), 174-179 (2013).

20. Malmberg, L. P., Rytila, P., Happonen, P. \& Haahtela, T. Inspiratory flows through dry powder inhaler in chronic obstructive pulmonary disease: age and gender rather than severity matters. Int J Chron Obstruct Pulmon Dis. 5, 257-262 (2010).

21. van der Palen, J. Peak inspiratory flow through diskus and turbuhaler, measured by means of a peak inspiratory flow meter (InCheck DIAL). Respir Med. 97(3), 285-289 (2003).

22. Duarte, A. G. et al. Spirometry Measurement of Peak Inspiratory Flow Identifies Suboptimal Use of Dry Powder Inhalers in Ambulatory Patients with COPD. Chronic Obstr Pulm Dis. 6(3), 246-255 (2019).

23. Cheng, S. L. et al. COPD in Taiwan: a National Epidemiology Survey. Int J Chron Obstruct Pulmon Dis. 10, 2459-2467 (2015).

24. Taylor, T. E., Holmes, M. S., Sulaiman, I., Costello, R. W. \& Reilly, R. B. Influences of gender and anthropometric features on inspiratory inhaler acoustics and peak inspiratory flow rate. Conf Proc IEEE Eng Med Biol Soc. 2015, 2227-2230 (2015).

\section{Author contributions}

Conception and design: S.Y.C. and J.Y.C. Analysis and interpretation: S.Y.C., C.K.H., C.J.Y. and J.Y.C. Data collection and analysis: S.Y.C., C.K.H., H.C.P., and J.Y.C. Drafting the manuscript for important intellectual content: S.Y.C., C.K.H and J.Y.C.

\section{Competing interests}

The authors declare no competing interests.

\section{Additional information}

Correspondence and requests for materials should be addressed to J.-Y.C.

Reprints and permissions information is available at www.nature.com/reprints.

Publisher's note Springer Nature remains neutral with regard to jurisdictional claims in published maps and institutional affiliations.

(c) (i) Open Access This article is licensed under a Creative Commons Attribution 4.0 International cc. License, which permits use, sharing, adaptation, distribution and reproduction in any medium or format, as long as you give appropriate credit to the original author(s) and the source, provide a link to the Creative Commons license, and indicate if changes were made. The images or other third party material in this article are included in the article's Creative Commons license, unless indicated otherwise in a credit line to the material. If material is not included in the article's Creative Commons license and your intended use is not permitted by statutory regulation or exceeds the permitted use, you will need to obtain permission directly from the copyright holder. To view a copy of this license, visit http://creativecommons.org/licenses/by/4.0/.

(c) The Author(s) 2020 